\title{
High-Quality Three-Dimensional Computed Tomography Angiography of Abdominal Viscera with Small Focal Spot, Low Tube Voltage, and Iterative Model Reconstruction Technique
}

\author{
Masafumi Uchida \\ Department of Radiology, Kurume University School of Medicine, Kurume, Japan \\ Email: krumf@med.kurume-u.ac.jp \\ Received 6 February 2015; accepted 23 February 2015; published 26 February 2015 \\ Copyright @ 2015 by author and Scientific Research Publishing Inc. \\ This work is licensed under the Creative Commons Attribution International License (CC BY). \\ http://creativecommons.org/licenses/by/4.0/ \\ (c) (i) Open Access
}

\begin{abstract}
Purpose: To evaluate the quality of three-dimensional (3D) CT angiography images of the abdominal viscera with small focal spot, low tube voltage, and iterative model reconstruction technique (IMR). Materials and Methods: Seven patients with suspected disease of the pancreatobiliary system had undergone CT with high-quality CTA protocol in the present study. There were 5 men and 2 women, ranging in age from 52 to 80 years (mean: 64 years). Results: Depiction of abdominal small artery, small portal vein was possible in all cases. In two cases that we were able to compare, it was superior to standard CTA in small vascular depiction in CTA made clearly in high quality protocol. Conclusions: Although the use of small focal spot, low tube voltage, and IMR can produce higher-quality images of abdominal vessels than standard CTA, this improvement is not significant at elevated radiation doses.
\end{abstract}

Keywords

CT, CT Angiography, Small Focal Spot, Low Tube Voltage, Iterative Model Reconstruction

\section{Introduction}

Medical three-dimensional (3D) angiography imaging can be conducted using a variety of techniques, including computed tomography (CT), magnetic resonance imaging (MRI), ultrasonography (US). Of these, 3D CTA uti- 
lizes data with the highest resolution to provide highest image quality and is therefore the most commonly used technique. Reliable 3D CTA rendering of the vascular anatomy of the abdomen aids tumor resection and laparoscopic surgery. An optimal 3D CTA image has a combination of the laparoscopic view and preoperative or intraoperative images. However, the capabilities and limitations of CTA have not been well characterized for the evaluation of diseases that involve small and very small arteries. Very small arteries are defined here as intraabdominal arteries with an average diameter of $\leq 1$ to $1.5 \mathrm{~mm}$, which is similar to that of intracranial arteries [1]. For example, present methods provide poor-quality depiction of hepatic sub segment and cystic arteries.

Abdominal CTA using a large field of view (FOV) provides inferior resolution compared to intra-arterial digital subtraction angiography (DSA). Therefore, sub-millimeter branches are relatively difficult to visualize on CTA due to their small diameter. This might be due to both mall veins and small arteries being difficult to depict by CTA, which hampers the definitive identification of small vessels [1].

Here, we discuss recent advances in CTA imaging techniques and 3D visualization of the abdomen, including scanning and image-reconstruction techniques.

\section{Materials and Methods}

\subsection{Patients}

Seven patients with a suspected abdominal disease underwent abdominal MDCT with high-quality CTA protocol in the present study. There were 5 men and 2 women, ranging in age from 52 to 80 years (mean: 64 years). Informed consent for participation in the study was obtained from each patient or guardian as part of the protocol approved by the Institutional Clinical Subpanel on Human Studies at our university hospital.

\subsection{Image Acquisition-High-Quality 3D CTA Imaging}

CT data are acquired using a 256-slice multi Detector CT system (Brilliance iCT; Philips Medical Systems, Eindhoven, the Netherlands)and a protocol for high-quality CTA imaging of the abdomen using small focal spots, low tube voltage $(100 \mathrm{kVp})$, and iterative model reconstruction techniques. Scanning is performed using a pitch of 0.60 , a 0.75 -sec scanning time per rotation, a table speed of $64.1 \mathrm{~mm} /$ rotation and detector configuration of $0.625 \times 128 \mathrm{~mm}, 100 \mathrm{kVp}$, and 230 or greater mAs depending on the size of the patient (Table 1). Contrastenhanced 3D CTA scans are conducted using a power injector (Auto Enhance A-50; Nemoto-Kyorin-Dou, Tokyo, Japan). During CTA, contrast medium (nonioniciodinated contrast medium: iodine concentration 350 $\mathrm{mg} \mathrm{I} / \mathrm{ml}$ ) is injected at a rate of $4 \mathrm{ml} / \mathrm{sec}$ via a 20 -gauge plastic IV catheter placed in an antecubital vein. The total volume administered is $630 \mathrm{mgI} / \mathrm{kg}$, including $20 \mathrm{ml}$ of saline $(\mathrm{NaCl} 0.9 \%)$, via flush injection. Images for each phase are obtained in the craniocaudal direction using a bolus-triggered technique with the cursor placed in the aorta and threshold set to $200 \mathrm{HU}$. The arterial phase is initiated $8 \mathrm{sec}$ after a $200 \mathrm{HU}$ aortic enhancement time. The portal venous and hepatic venous phase CT scans are obtained at 28 and $40 \mathrm{sec}$ after the arterial phase scan, respectively [2].

Table 1. Scan protocol of High-quality 3D CTA imaging.

\begin{tabular}{cc} 
Voltage $(\mathrm{kVp})$ & $100 \mathrm{kVp}$ \\
\hline Tube current (mA) & $230-680 \mathrm{~mA}$ \\
Gantry revolution time (sec) & $0.75 \mathrm{sec}$ \\
Pitch & 0.60 \\
Field of view (mm) & $320-340 \mathrm{~mm}$ \\
Reconstruction algorithm & Full iterative reconstruction IMR \\
Focus size (mm) & $0.6 \times 0.7$ mm (small focal spot) \\
Matrix & $\underline{512 \times 512}$ \\
\hline
\end{tabular}

\footnotetext{
${ }^{*}$ Contrast enhancement: contrast medium $350 \mathrm{mgI} / \mathrm{mL}, 1.7 \mathrm{~mL} / \mathrm{kg}$. Injection rate: $4 \mathrm{~mL} / \mathrm{sec}$. The arterial phase was initiated at 8 seconds after a 200
} HU aortic enhancement time using the bolus-tracking technique with a $20 \mathrm{ml}$ saline ( $\mathrm{NaCl} 0.9 \%$ ) flush injection. 


\subsection{Image Post-Processing and Interpretation}

CT data for each phase were retrospectively reconstructed with iterative model reconstruction (IMR) at 0.6251.25-mm slice thickness per section. Raw data were automatically transferred to a workstation (ZIOSTATION2; Ziosoft, Tokyo, Japan) in a $512 \times 512$ pixel format via Ethernet. The 3- or 4-phase source two-dimensional CT images were reviewed and analyzed for 3D CTA reconstruction using the workstation.

\section{Results}

High-quality CTA protocol was performed for all patients enrolled in the study. Two cases were performed in standard protocol CT in this within a half year. Depiction of abdominal small artery, small portal vein was possible in all cases (Figures 1-3). In two cases that we were able to compare, it was superior to standard CTA in small vascular depiction in CTA made clearly in high quality protocol (Figure 1).

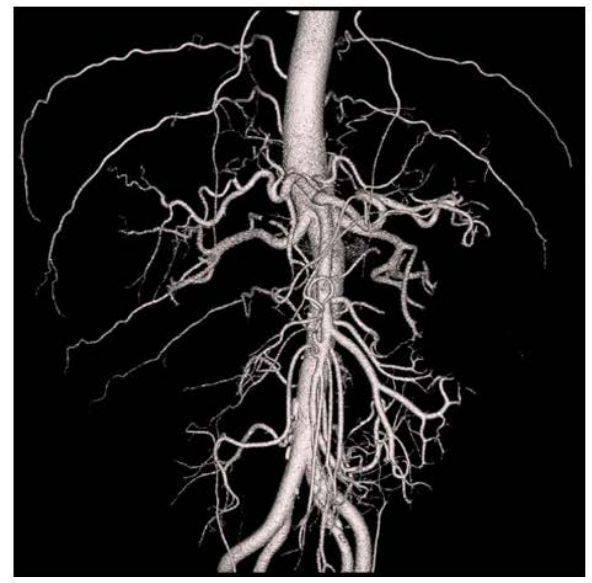

(a)

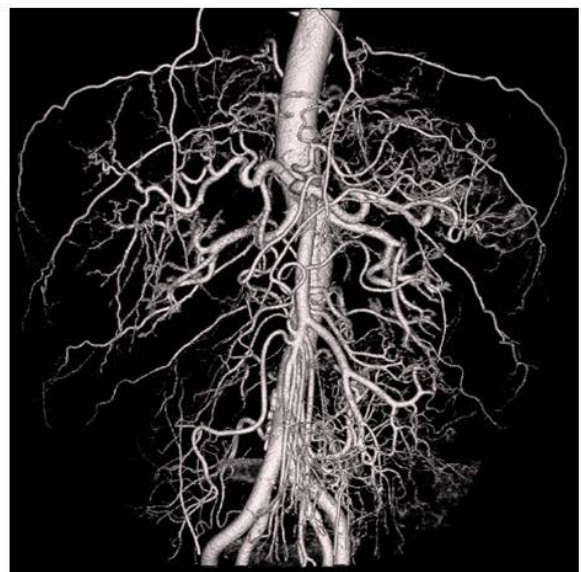

(b)

Figure 1. A 57-year-old woman with intraductal papillary mucinous neoplasm (IPMN). Two CT examinations were performed on follow-up every three months. The first was a standard CT scan (large focal spot, $120 \mathrm{kVp}$, FBP reconstruction), and the second a high-quality CT protocol scan. (a) CT arteriography from standard CT protocol; and (b) CT arteriography from the high-quality CT protocol using same opacity curve. High-quality CT arteriography (b) clearly shows the small subsegmental hepatic arteries of the liver and other small abdominal viscera artery branches. Visibility and sharpness of very small abdominal arteries were improved with high-quality CT arteriography.

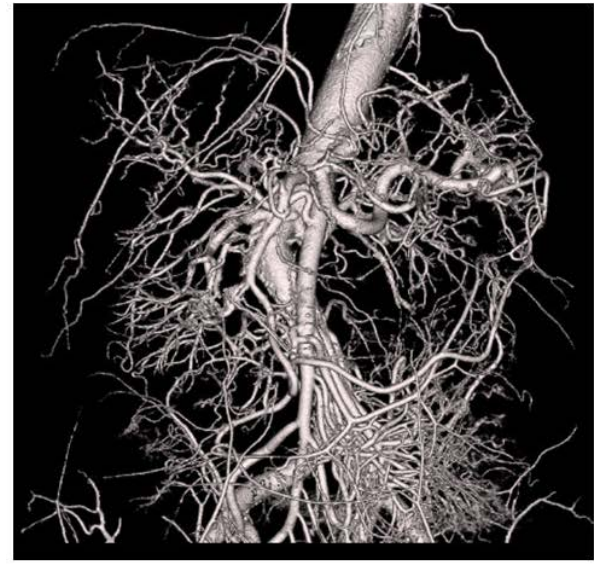

(a)

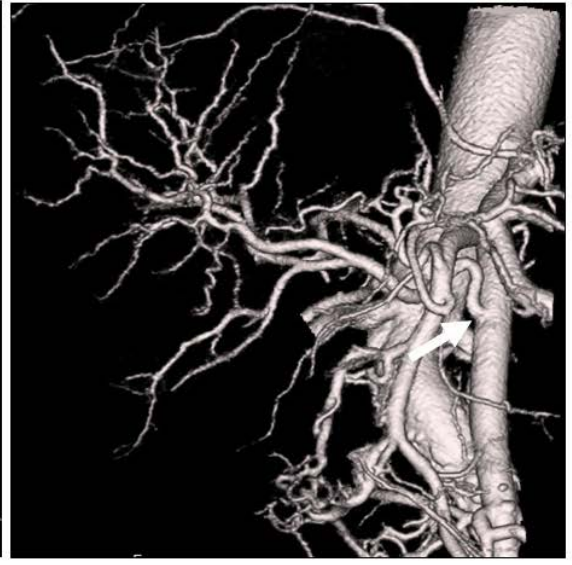

(b)

Figure 2. A 46-year-old woman with gallbladder adenomyomatosis. (a) The high-quality CT arteriography clearly shows small arteries of the abdominal viscera; (b) This CT arteriography image only depicts the right hepatic artery. CT arteriography clearly shows the right hepatic artery arising from the superior mesenteric artery (white arrow) and small cystic artery and sub segment hepatic arteries. 


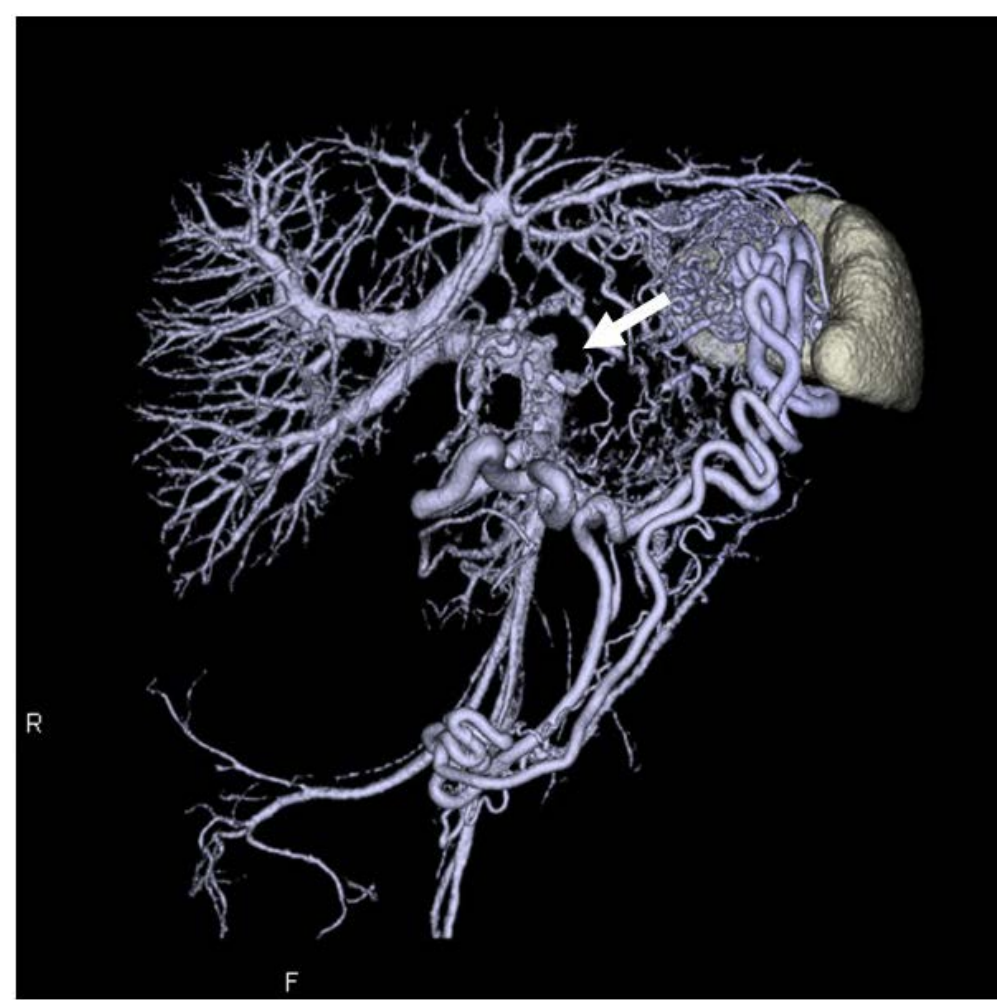

Figure 3. A 62-year-old man with pancreatic cancer. High-quality CT portography clearly depicting the vascular structure and showing complete occlusion of the splenic vein (white arrow). CT portography clearly shows small subsegmental portalveins of the liver and other very small abdominal portal branches. This image confirms the formation of an eminent collateral vein.

\section{Discussion}

The recent introduction of MDCT scanners for abdominal imaging has enabled the acquisition of optimal dynamic images with high temporal and $z$-axis resolution. MDCT also enables the comprehensive evaluation of CTA data. In abdominal vessels, 3D CTA is now a suitable diagnostic procedure for disorders of the aorta and major aortic branches, and 3D CT portography and venography are effective in depicting hepatic portal venous anatomy [2] [3]. Our high-quality CTA protocol produced higherquality vessel images than a standard CT scan (large focal spot, $120 \mathrm{kVp}$, FBP reconstruction), and using this protocol, imaging of sub-millimeter branches might also be possible (Figures 1-3).

The size of the focal spot is a critical parameter in many X-ray imaging systems, including projection radiography and CT. Many CT tubes have two focalspot sizes, often referred to as "small" and "large". To produce $\mathrm{X}$-ray images with minimum blur, a small focal spot is preferable, and CT is an example of a setup where a small focal spot is preferable for producing a high-detail image with high spatial resolution. Conventionally, body structures for which good low-contrast resolution is essential require scanning with a large focal spot and high power, whereas high-resolution images with thin slices require a small focal spot. The recent advent of high-output X-ray tubes for MDCT scanners has enabled an increase in the upper limit of mA for small focal spot sizes [4] [5]. Regarding CT scans, automatic exposure control systems (AECs) have become increasingly common. However, AEC does not have a system that guarantees a similar intensity to that of the large and small focal spots. We therefore coordinated tube current according to patient size.

CT images are a display of the degree of attenuation that has occurred when the X-ray beam penetrates the body, an effect known as the linear attenuation coefficient. Measurement of the density of this attenuated beam is assigned a CT number (HU), which is related to this linear attenuation coefficient. The $\mathrm{kVp}$ selection has a direct effect on these linear coefficient values. Lowering the peak kilovoltage setting (80 - $100 \mathrm{kVp})$ is an effective method of reducing radiation dose while also increasing vascular enhancement, as the attenuation of iodine-based contrast agents increases with reduced X-ray energy distribution due to the high relative atomic 
number of iodine and the k-edge in these energy levels [6].

Recently, several manufacturers have introduced new CT reconstruction algorithms based on iterative rather than traditional filtered back-projection (FBP) reconstruction. Iterative reconstruction (IR) algorithms provide better image quality than FBP on CTA, mostly due to reduced image noise and thus improved contrast to noise ratio (CNR) [7]. These observations suggest that IR algorithms may be more efficient than FBP, and manufacturers have proposed that they decrease radiation dose. These observations suggest that IR algorithms might reduce the dosage more efficiently than FBP reconstruction, while also maintaining equivalent image quality. However, the conventional IR method is a hybrid, in which the IR and FBP techniques are combined.

In contrast, IMR is a new and full IR technique. IMR method is the newest IR method and is required to ensure complete reconstruction. Further, this method provides greater noise reduction than hybrid IR and improves both spatial and contrast resolution [8]. The IR method is also very effective in reducing noise with low tube voltage settings as well as radiation dose exposure.

High-quality 3D CTA imaging opens up the possibility of aligning true and detailed anatomical information of the abdominal vascular system with spatial data provided by MDCT.

\section{Conclusion}

Although a small focal spot, low tube voltage, and use of IMR techniques can produce higher-quality images of abdominal vessels than standard CTA, this improvement is not significant at elevated radiation doses.

\section{References}

[1] Villablanca, J.P., Rodriguez, F.J., Stockman, T., et al. (2007) MDCT Angiography for Detection and Quantification of Small Intracranial Arteries: Comparison with Conventional Catheter Angiography. American Journal of Roentgenology, 188, 593-602. http://dx.doi.org/10.2214/AJR.05.2143

[2] Uchida, M. (2014) Recent Advances in 3D Computed Tomography Techniques for Simulation and Navigation in Hepatobiliary Pancreatic Surgery. Journal of Hepato-Biliary-Pancreatic Sciences, 21, 239-245. http://dx.doi.org/10.1002/jhbp.82

[3] Ichikawa, T., Motosugi, U., Morisaka, H., et al. (2012) Optimal Iodine Dose for 3-Dimensional Multidetector-Row CT Angiography of the Liver. European Journal of Radiology, 81, 2450-2455. http://dx.doi.org/10.1016/j.ejrad.2011.06.022

[4] Sprawls, P. (1992) CT Image Detail and Noise. RadioGraphics, 12, 1041-1046. http://dx.doi.org/10.1148/radiographics.12.5.1529128

[5] Seeram, E. (2009) Computed Tomography: Physical Principles, Clinical Applications, and Quality Control/Euclid Seeram, n.p.: Edinburgh: Saunders/Elsevier, c2009.

[6] Marin, D., Nelson, R.C., Samei, E., et al. (2009) Hypervascular Liver Tumors: Low Tube Voltage, High Tube Current Multidetector CT during Late Hepatic Arterial Phase for Detection-Initial Clinical Experience. Radiology, 251, 771779. http://dx.doi.org/10.1148/radiol.2513081330

[7] Utsunomiya, D., Weigold, W.G., Weissman, G., et al. (2012) Effect of Hybrid Iterative Reconstruction Technique on Quantitative and Qualitative Image Analysis at 256-Slice Prospective Gating Cardiac CT. European Radiology, 22, 1287-1294. http://dx.doi.org/10.1007/s00330-011-2361-6

[8] Pickhardt, P.J., Lubner, M.G., Kim, D.H., et al. (2012) Abdominal CT with Model-Based Iterative Reconstruction (MBIR): Initial Results of a Prospective Trial Comparing Ultralow-Dose with Standard-Dose Imaging. American Journal of Roentgenology, 199, 1-9. http://dx.doi.org/10.2214/AJR.12.9382 
Scientific Research Publishing (SCIRP) is one of the largest Open Access journal publishers. It is currently publishing more than 200 open access, online, peer-reviewed journals covering a wide range of academic disciplines. SCIRP serves the worldwide academic communities and contributes to the progress and application of science with its publication.

Other selected journals from SCIRP are listed as below. Submit your manuscript to us via either submit@scirp.org or Online Submission Portal.
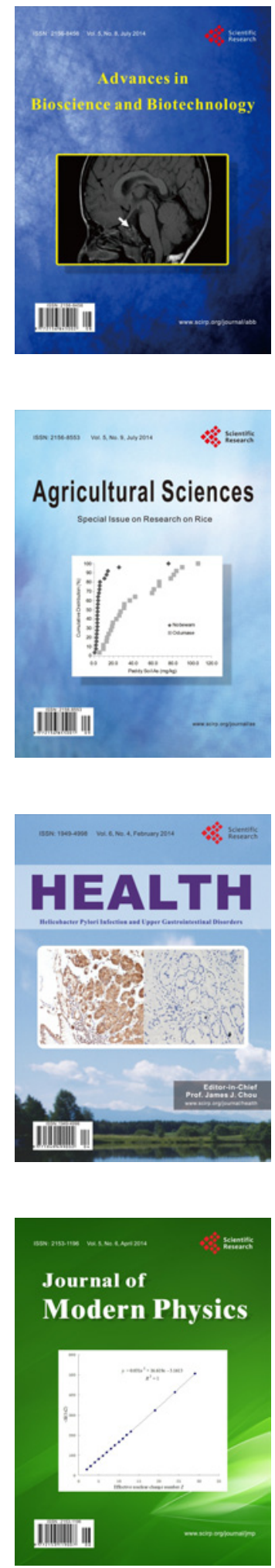
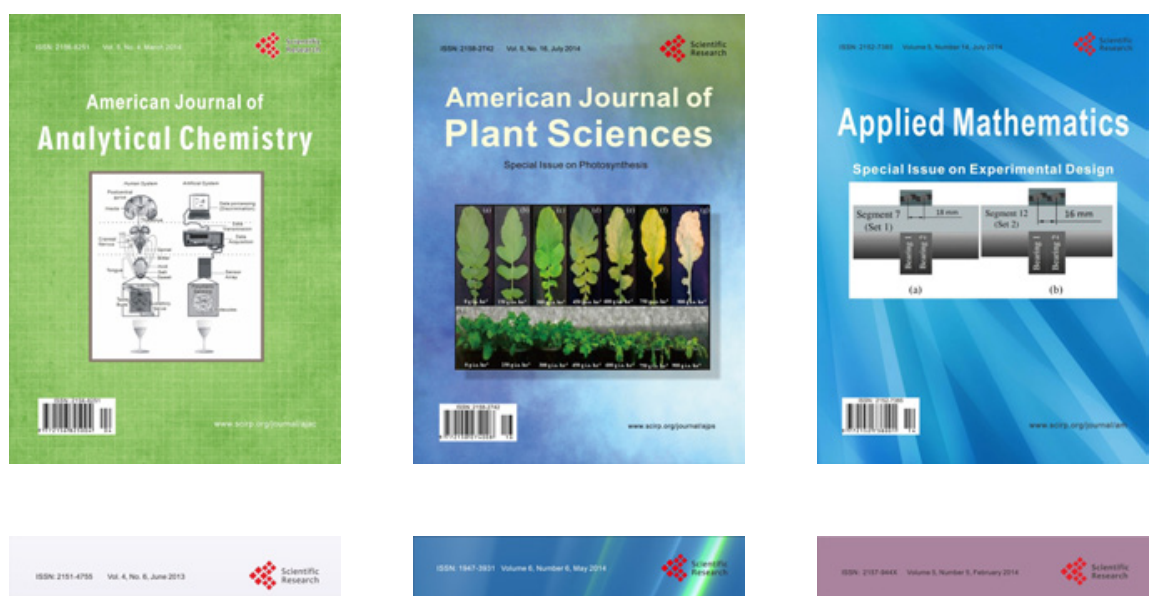

Creative Education
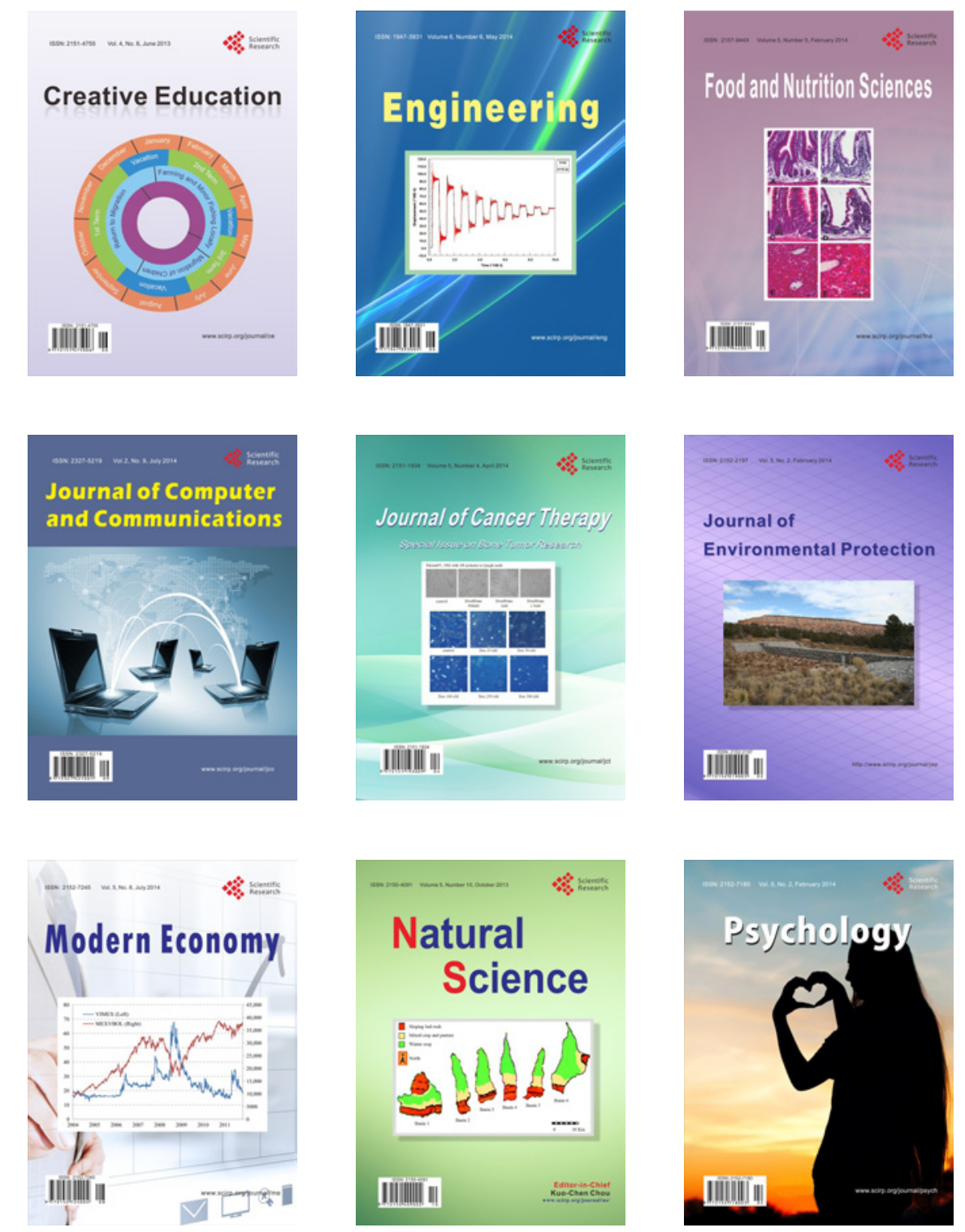